Radial and Nonradial Pulsations as Probes of Stellar Physics

ASP Conference Series, Vol. 259, 2002

C. Aerts, T.R. Bedding, \& J. Christensen-Dalsgaard, eds.

\title{
Time Resolved Spectroscopy of the Pulsating sdB Star PG 1605+072 ${ }^{1}$
}

\author{
S. J. O'Toole, T. R. Bedding
}

School of Physics, University of Sydney, Australia

H. Kjeldsen

Theoretical Astrophysics Center, Aarhus University, Denmark

T. Dall, D. Stello

Institute of Physics and Astronomy, Aarhus University, Denmark

\begin{abstract}
We present analysis of rapid spectroscopy of PG 1605+072, a pulsating sdB star that is believed to have evolved off the extreme horizontal branch. Using a cross-correlation technique on five Balmer lines $(\mathrm{H} \beta$ to $\mathrm{H} 8)$, we have been able to study the Doppler variations in this star over one year. Preliminary results for about $25 \%$ of the time-series were presented by O'Toole et al. (2000). We find evidence for amplitude variation between 1999 and 2000 . We have also examined of equivalent widths of the Balmer lines looking for variation. Preliminary results are intriguing, with the amplitude of the equivalent width oscillations strongly dependent on wavelength.
\end{abstract}

\section{Introduction}

The discovery of multi-mode pulsations in hot subdwarfs has opened up the opportunity to probe the interiors of these stars with the tools of asteroseismology. A review of the properties and current status of observations of pulsating sdBs can be found in Kilkenny (these proceedings), and the theoretical aspects in Charpinet (these proceedings).

Of all the pulsating sdB stars known, PG $1605+072$ has the highest amplitudes (around $30 \mathrm{mmag}$ in $V)$ and longest periods $(\sim 500 \mathrm{~s})$, making it an ideal target for time-resolved spectroscopy. The star also has the lowest gravity of the pulsating sdBs and it is likely that it has evolved off the extreme horizontal branch. The paper by Woolf et al. (these proceedings) details observations of PG $1605+072$ with resolution better than $1 \AA$.

\footnotetext{
${ }^{1}$ Based on observations made with the Danish $1.54 \mathrm{~m}$ telescope at ESO, La Silla, Chile, and on observations made with the Nordic Optical Telescope, operated on the island of La Palma jointly by Denmark, Finland, Iceland, Norway and Sweden in the Spanish Observatorio del Roque de Los Muchachos of the Instituto de Astrofisica de Canarias.
} 


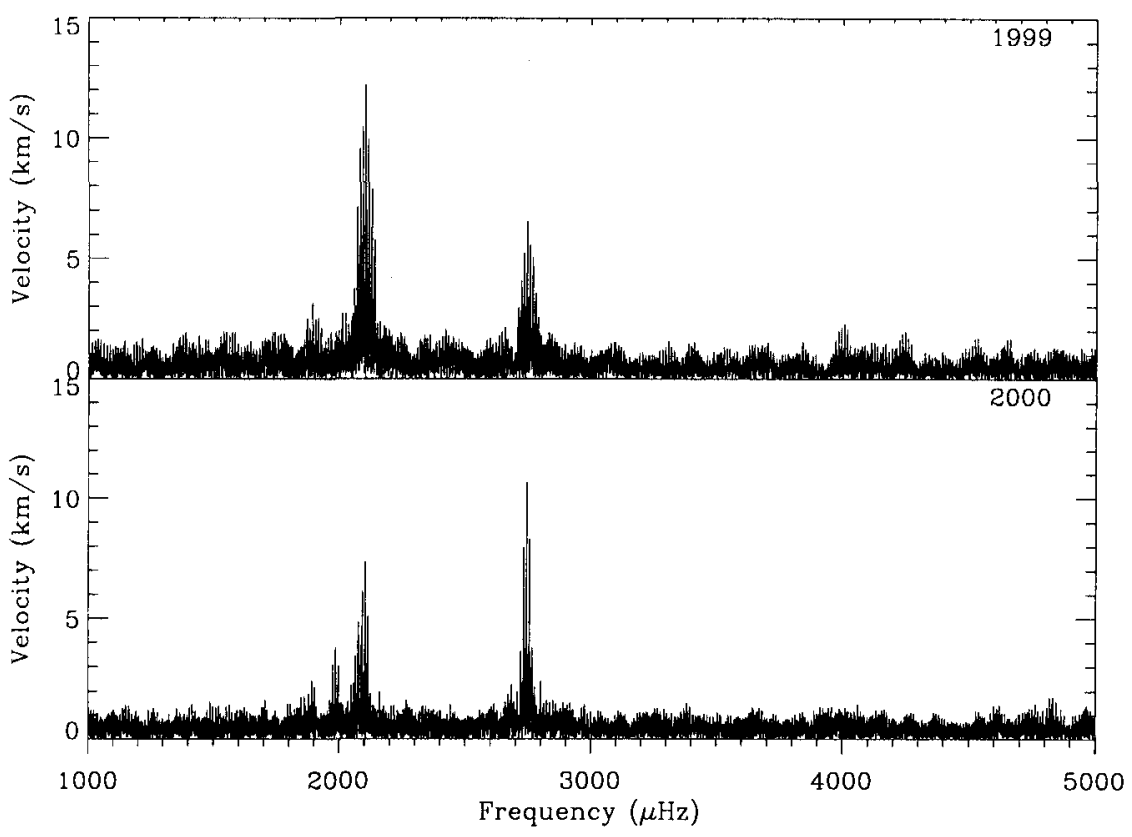

Figure 1. Comparison of 1999 and 2000 observations. It is uncertain whether the apparent change in amplitude is due to beating between closely spaced modes or a change in oscillation amplitude.

\section{Observations}

The observations of PG $1605+072$ were made on 11 nights over a 16-day period in May 2000, using the DFOSC spectrograph on the Danish $1.54 \mathrm{~m}$ telescope at La Silla, Chile and the ALFOSC spectrograph mounted on the $2.56 \mathrm{~m}$ Nordic Optical Telescope on La Palma in the Canary Islands.

After basic reductions were done using IRAF, we used a cross-correlation technique to determine velocity variations for five Balmer lines $(\mathrm{H} \beta$ to $\mathrm{H} 8$ ). The short oscillation periods of PG $1605+072$ allow us to use the Balmer lines themselves as wavelength calibrators, since any drifts in the instruments will be on much longer time-scales. We have also begun examining the equivalent width (EW) variations using the method described by Baldry et al. (1999). The quality of our velocity and EW measurements vary from night to night, so we have applied weights based on the internal scatter (Kjeldsen \& Frandsen, 1992).

\section{Main results}

The velocity amplitude spectra for each Balmer line was calculated, and the noise level for each found to be $\sim 600 \mathrm{~m} \mathrm{~s}^{-1}$. O'Toole et al. (2000) found a possible 


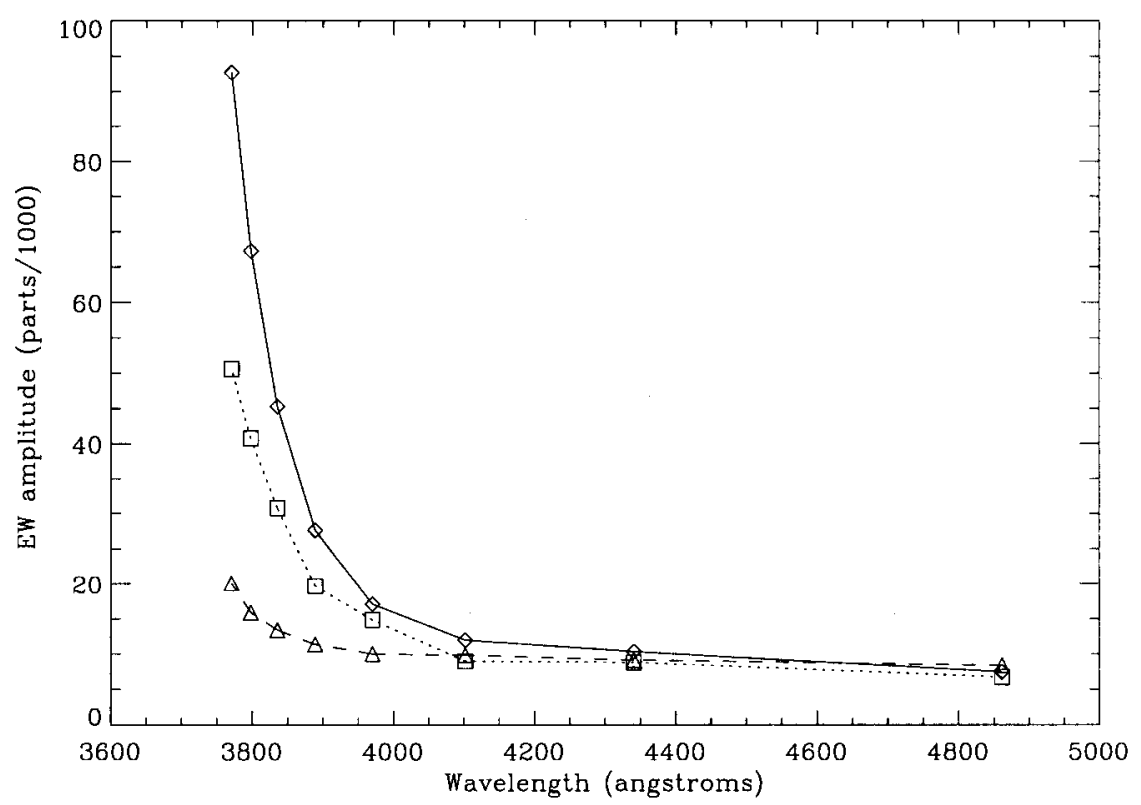

Figure 2. Balmer line EW amplitude as a function of wavelength. The solid line with diamonds represents the $2742 \mu \mathrm{Hz}$ mode, the dotted line with squares represents the $2102 \mu \mathrm{Hz}$ mode and the dashed line with triangles is a model with an EW variation of $\sim 210 \mathrm{~K}$.

wavelength dependence of the velocity amplitudes in PG $1605+072$, but we find no evidence of such a dependence.

The amplitude spectra of the combination of all Balmer line time-series for both our 1999 and 2000 observations are shown in Fig. 1. There is an apparent change in amplitude between the two sets of observations. At present we are unsure if this is a change in oscillation amplitude or simply beating between many closely spaced frequencies. Kilkenny et al. (1999) found many low amplitude, very closely spaced frequencies in the region of the two dominant peaks $(\sim 2.10 \mathrm{mHz}$ and $\sim 2.74 \mathrm{mHz})$, pointing to the latter explanation.

We find a dramatic variation in the equivalent-width amplitudes with wavelength, as shown in Fig. 2. The two strongest modes are plotted as a solid line $(2742 \mu \mathrm{Hz})$ and as a dotted line $(2102 \mu \mathrm{Hz})$. To explain this increase, we have used synthetic sdB spectra (courtesy of Uli Heber) at different effective temperatures $(27500$ to $37500 \mathrm{~K})$ to measure the expected EW variations. This assumes that the EW is only dependent on temperature. We found the EW at two different temperatures, took the difference and then determined the fractional EW. We find that this value (which is equivalent to an oscillation amplitude) is approximately proportional to temperature, meaning that we can scale the variations to the approximate temperature expected from photometry $(\sim 210 \mathrm{~K})$. 
This is shown as the dashed line in Fig. 2. It is clear that this is not the complete explanation.

\section{Conclusions and future work}

We have found evidence for amplitude variability over time in PG 1605+072. A time-series with better frequency resolution will be able to confirm this. A campaign of this type is currently being organised.

The equivalent-width variations of the Balmer lines have a strong wavelength dependence. We have shown it is not exclusively a temperature effect, but have not yet taken into account changes in gravity. An effort to do this will be part of continuing analysis.

\section{References}

Baldry, I.K., Viskum, M., Bedding, T.R., Kjeldsen, H., \& Frandsen, S. 1999, MNRAS, 302, 381

Kilkenny, D., Koen, C., O'Donoghue, D., van Wyk, F., Larson, K.A., Shobbrook, R., Sullivan, D.J., Burleigh, M.R., Dobbie, P.D., \& Kawaler, S.D. 1999, MNRAS, 303, 525

Kjeldsen, H. \& Frandsen, S. 1992, PASP, 104, 413

O'Toole, S.J., Bedding, T.R., Kjeldsen, H., Teixeira, T.C., Roberts, G., van Wyk, F., Kilkenny, D., D'Cruz, N. \& Baldry, I.K. 2000, ApJ, 537, L53

\section{Discussion}

W. Weiss : To what extent could the problem with continuum definition explain the EW effects?

S. O'Toole : This may certainly be a problem for higher order Balmer lines ( $\mathrm{H} 9$ to $\mathrm{H} 11$, even $\mathrm{H} 8$ ) but the effect is evident in $\mathrm{H} \delta$ and $\mathrm{H} \epsilon$, where the continuum should be well defined. The actual effect is what I am currently trying to investigate using the models.

T. Medupe : Have you considered limb-darkening in this wavelength region? Limb darkening for A stars and pulsating white dwarfs increases rapidly with decreasing wavelength and has been used to explain the high amplitudes in white dwarfs.

S. O'Toole : I have not considered limb darkening... yet. However, if you would like to work with me on the modelling, I would like to explore all possibilities. 